\title{
Altered gene expression and spongiotrophoblast differentiation in placenta from a mouse model of diabetes in pregnancy
}

\author{
J. M. Salbaum • C. Kruger • X. Zhang • \\ N. Arbour Delahaye • G. Pavlinkova $\cdot$ D. H. Burk • \\ C. Kappen
}

Received: 2 November 2010 / Accepted: 4 March 2011 /Published online: 14 April 2011

(C) Springer-Verlag 2011

\begin{abstract}
Aims/hypothesis Pregnancies complicated by diabetes have a higher risk of adverse outcomes for mothers and children, including predisposition to disease later in life, e.g. metabolic syndrome and hypertension. We hypothesised that adverse outcomes from diabetic pregnancies may be linked to compromised placental function, and sought to identify cellular and molecular abnormalities in diabetic placenta.
\end{abstract}

Electronic supplementary material The online version of this article (doi:10.1007/s00125-011-2132-6) contains supplementary material, which is available to authorised users.

\author{
J. M. Salbaum • N. A. Delahaye \\ Laboratory of Regulation of Gene Expression, \\ Pennington Biomedical Research Center, \\ Baton Rouge, LA, USA \\ C. Kruger $\cdot$ X. Zhang $\cdot$ C. Kappen $(\bowtie)$ \\ Department of Maternal Biology, \\ Pennington Biomedical Research Center, \\ 6400 Perkins Road, \\ Baton Rouge, LA 70808, USA \\ e-mail: claudia.kappen@pbrc.edu \\ G. Pavlinkova \\ Department of Pediatrics, University of Nebraska Medical Center, \\ Omaha, NE, USA \\ D. H. Burk \\ Cell Biology and Bioimaging Core, \\ Pennington Biomedical Research Center, \\ Baton Rouge, LA, USA \\ Present Address: \\ G. Pavlinkova \\ Institute of Biotechnology of the Academy of \\ Sciences of the Czech Republic, \\ Prague, Czech Republic
}

Methods Using a mouse model of diabetic pregnancy, placental gene expression was assayed at mid-gestation and cellular composition analysed at various stages. Genome-wide expression profiling was validated by quantitative PCR and tissue localisation studies were performed to identify cellular correlates of altered gene expression in diabetic placenta.

Results We detected significantly altered gene expression in diabetic placenta for genes expressed in the maternal and those expressed in the embryonic compartments. We also found altered cellular composition of the decidual compartment. In addition, the junctional and labyrinth layers were reduced in diabetic placenta, accompanied by aberrant differentiation of spongiotrophoblast cells.

Conclusions/interpretation Diabetes during pregnancy alters transcriptional profiles in the murine placenta, affecting cells of embryonic and maternal origin, and involving several genes not previously implicated in diabetic pregnancies. The molecular changes and abnormal differentiation of multiple cell types precede impaired growth of junctional zone and labyrinth, and of placenta overall. Regardless of whether these changes represent direct responses to hyperglycaemia or are physiological adaptations, they are likely to play a role in pregnancy complications and outcomes, and to have implications for developmental origins of adult disease.

Keywords Diabetic placenta - Diabetic pregnancy · Gene expression profiling . Gene regulation . Hyperglycaemia · Junctional layer - Maternal diabetes . Placental insufficiency . Spongiotrophoblast . Transcription factors 


\author{
Abbreviations \\ DAVID Database for Annotation, Visualization and Inte- \\ grated Discovery \\ FOX Forkhead box \\ HIF1 Hypoxia-inducible factor 1 \\ MGI Mouse Genome Informatics \\ NFkB Nuclear factor $\kappa B$ \\ TFBS Transcription factor binding site \\ TPBPA Trophoblast-specific protein alpha
}

\section{Introduction}

Diabetes in pregnancy is a well established risk factor for diabetic embryopathy, a spectrum of congenital malformations that include heart defects and neural tube defects [13]. Exposure to maternal diabetes during pregnancy also predisposes to disease later in life, e.g. metabolic syndrome, obesity, diabetes and hypertension $[4,5]$. While mechanistic studies on developmental origins of adult disease are still in early stages, substantial progress has been made in identifying causes of the congenital abnormalities. Thus in rodent models, metabolic perturbations and nutrient deficiencies have been demonstrated, including perturbed prostaglandin metabolism and inositol deficiency. Oxidative stress and hypoxia have also been documented as causes [6], and antioxidant administration reduced the incidence of fetal malformations. In addition to increased apoptosis, developmental regulators [7] and signalling pathways [8, 9] are perturbed in exposed embryos, implicating these mechanisms in abnormal morphogenesis and congenital malformations in diabetic embryopathy.

There is, however, little knowledge about the basis for increased disease susceptibility later in life in progeny exposed to maternal diabetes. Once an embryo has escaped the critical time window of vulnerability to congenital defects, it nevertheless remains exposed to the diabetic environment. We have recently shown that this leads to reduction of intrauterine growth during later stages of gestation [10], possibly due to hyperglycaemia-induced changes in the placenta. It has been proposed that placental changes could be central to developmental programming of disease susceptibility of the offspring [11, 12], but the underlying molecular mechanisms are unknown. Altered placental weight has been reported for diabetic rats [13], and for human type 1 [14] and gestational diabetic [15] pregnancies. Human type 1 diabetic placenta undergoes increased angiogenesis and exhibits vascular dysfunction, mediated through vascular endothelial growth factor and nitric oxide in early pregnancy, and through hyperinsulinaemia in the later trimesters [16]. Furthermore, enrichment of glycogen [17] and excess lipid accumulation [18, 19] have been described in rat diabetic placenta.

To identify the molecular basis of placental abnormalities in diabetic pregnancies in the mouse, we used a streptozotocin-induced model of diabetes. Our results demonstrate that altered gene expression at mid-gestation precedes cellular and structural abnormalities in diabetic mouse placenta at later stages.

\section{Methods}

Animals All experiments were conducted in accordance with the United States Public Health Service Policy on Humane Care and Use of Laboratory Animals (http:// grants1.nih.gov/grants/olaw/references/phspol.htm) in a facility approved by the American Association for Accreditation of Laboratory Animal Care. Diabetes was induced in 7- to 9-week-old FVB female mice (Charles River Laboratories, Wilmington, MA, USA) by two i.p. injections of streptozotocin $(100 \mathrm{mg} / \mathrm{kg}$ body weight) and matings were set up as described previously [9]. Dams whose glucose levels in tail vein blood (Contour Glucometer; Bayer, Tarrytown, NY, USA) exceeded $13.9 \mathrm{mmol} / \mathrm{l}$ were considered diabetic; average glucose levels were $8.22 \mathrm{mmol} / 1( \pm 1.0 \mathrm{SD})$ before streptozotocin treatment, $18.722 \pm 4.4 \mathrm{mmol} / \mathrm{l}$ in diabetic dams on the day of mating and $29.33 \pm 3.9 \mathrm{mmol} / \mathrm{l}$ on the day of placenta removal (from dams at gestational day E10.5, counting the day of detection of vaginal plug as E0.5).

Microarray analysis Whole placentae were dissected at E10.5; only specimens associated with morphologically normal embryos were used. Decidua were removed from the uterus, and the yolk sac and embryos were dissected out; placenta samples consisted of embryonic and maternal tissue. Placentae were processed as individual samples following published methods [9]. Samples were hybridised to 10 Affymetrix Mouse 4302.0 arrays (Affymetrix, Santa Clara, CA, USA), which were scanned using a scanner (GeneChip3000, Affymetrix). Quality control was done with GeneChip Operating Software (Affymetrix) and statistical analyses were performed as previously described for embryos [9]. The primary data files are available at the NCBI Gene Expression Omnibus repository (accession number GSE 28277).

Quantitative real-time PCR Quantitative real-time PCR was performed as described [9] using the ABI Prism 7900HT device (Life Technologies, Carlsbad, CA, USA). Primers (electronic supplementary material [ESM] Table 1) were positioned to span exon-exon junctions and amplicons were designed to originate from a different transcript 
region than that detected by microarray probes. Complementary DNA samples were derived from individual E10.5 placentae from different diabetic and control dams than those from which samples were assayed on microarrays. For normalisation, we used measurement of polymerase epsilon 4 (Pole4, also known as Pole4) expression; Pole4 expression proved to be unaffected by maternal diabetes on the arrays.

Annotation for molecular function and mutant mouse phenotypes Molecular function attributes were based on Gene Ontology annotation (NetAffx www.affymetrix.com/ analysis/netaffx/index.affx), supplemented with information from PubMed and Database for Annotation, Visualization and Integrated Discovery (DAVID) (http://david.abcc. ncifcrf.gov/), which was also used to examine for enrichment of specific categories. Information on mouse mutants was obtained from Mouse Genome Informatics (MGI) (www.informatics.jax.org; accessed 20 December, 2010). For transcription factor binding site (TFBS) prediction, Whole Genome rVISTA (http://genome.lbl.gov/vista/index. shtml) was used to identify motifs that are conserved between mouse and human and are over-represented in the $5 \mathrm{~kb}$ upstream regions of diabetes-affected genes, relative to all $5 \mathrm{~kb}$ upstream regions in the genome $(p<0.005)$.

Histology Placentae from normal and diabetic pregnancies were embedded in paraffin using a Shandon Excelsior tissue processor (Thermo, Waltham, MA, USA) and an embedder (Histocentre 3, Thermo), and sectioned at a thickness of $6 \mu \mathrm{m}$. Deparaffinisation and staining with haematoxylin/eosin was done in a Special Stainer device (ST5020; Leica Microsystems, Bannockburn, IL, USA), followed by coverslipping (CV5030; Leica). Slides were scanned on a digital pathology station (NDP Nanozoomer; Hamamatsu, Bridgewater, NJ, USA).

In situ hybridisation In situ hybridisations were performed as published [20] to either $20 \mu \mathrm{m}$ frozen sections or $6 \mu \mathrm{m}$ sections of paraffin-embedded tissues. Processing of slides was done together for diabetic and control samples in the same vessel for all fixation, wash, hybridisation, incubation and development steps. Plasmids for probe generation were obtained from Open Biosystems (Huntsville, AL, USA). For comparison of control and diabetic tissues, photography was done with the same magnification and exposure.

Cell counting and statistical analysis All sections were from the middle of the respective placentae. The sizes of placental compartments were determined as the respective area fractions in ImageJ (National Institutes of Health, Bethesda, MD, USA; http://rsbweb.nih.gov/ij/download. html, accessed 14 March 2011). Cell cluster counting was done by selecting the labyrinth only and counting of pixels with Tpbpa signal above a uniform threshold was performed. The validity of these methods was confirmed manually by two individuals, independently. For each placenta, data from ten sections were averaged and adjusted for size of the whole placenta. We included six placentae each in the control and diabetic groups for gestational day E15.5 and three specimens per group were used for E18.5. Each placenta came from a different pregnancy. Embryo and placenta size were averaged to each dam before averaging for the group. Statistical analysis was done by two-sided $t$ test.

Mouse model of diabetic pregnancy We employed the well-established streptozotocin-induced mouse model of diabetes, using the FVB inbred strain of mice. Embryos and placentae were collected at embryonic day 10.5 (E10.5) because at this stage, neural tube defects are easily detectable. Samples associated with malformed embryos were excluded from the analysis. Control embryos ( $n=65$ embryos from nine dams) had a crownrump length of $5.17 \pm 0.34 \mathrm{~mm}$ (mean $\pm \mathrm{SD}$ ). In diabetesexposed embryos ( $n=97$ embryos from 13 dams) crownrump length was $5.07 \pm 0.39 \mathrm{~mm}$. Differences were not statistically significant $(p=0.54)$. Placentae looked normal at this time point, but they differed $(p=0.0038)$ in size, with control placenta $(n=31$ placentae from seven dams) having a wet weight of $42.87 \pm 2.01 \mathrm{mg}$ and diabetic placentae ( $n=61$ placentae from nine dams) weighing $37.92 \pm 3.96 \mathrm{mg}$. From five control dams and five diabetic dams, we collected one placenta each of a weight close to the mean for the respective group and performed ten independent microarray assays (Affymetrix 430 2.0). Separate placentae were used for validation studies by quantitative real-time PCR. There were no significant differences in litter size at any of the gestational points investigated, except at E18.5, where control dams on average had $9.8 \pm 2.31$ embryos and diabetic dams had $8.1 \pm$ 3.04 progeny.

Altered gene expression profiles in placentae from diabetic dams From the 45,401 probe sets represented on the microarrays, we identified 158 genes where expression levels in placentae differed significantly between the experimental and control groups by more than 1.5 -fold (ESM Table 2). Of these 158 genes, 73 (46.2\%) were expressed at lower levels and $85(53.8 \%)$ genes at higher levels in diabetic placentae (Fig. 1a). This is in contrast to the diabetes-exposed embryo (Fig. 1b), where more than $85 \%$ of the differentially expressed genes were decreased in expression compared with controls [9]. Even where identical genes exhibited differential expression on embryo and placenta microarrays under conditions of maternal 

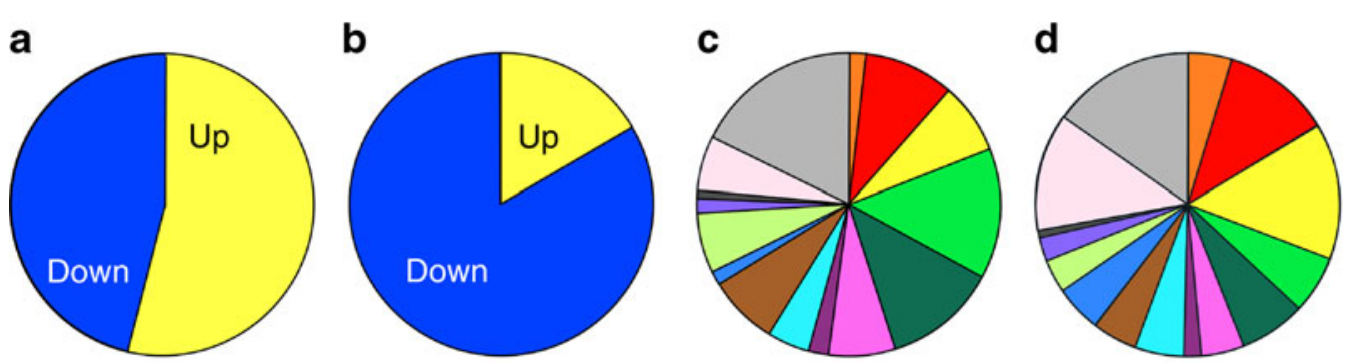

DNA binding/chromatin Transcription factors Signal transduction Cell surface, receptors, growth factors Extra-cellular matrix, adhesion Metabolism Lipid metabolism Cytoskeleton Protein processing RNA binding Transporters/channels Cell cycle/apoptosis Metal ion homeostasis Other Other

Fig. 1 Comparison of gene expression profiles in placentae and embryos under conditions of maternal diabetes. The data for placentae are from this report; the data for embryos are from previously published material [9]. Direction of change for (a) placenta genes and

diabetes (ESM Table 3), 62.5\% of these displayed discordant regulation, reflecting opposite directions of misregulation in embryo and placenta. These results underscore the notion that responses of placenta and embryo to maternal diabetes are fundamentally different at the molecular level, probably implicating different molecular mechanisms of regulation.

This is also reflected in the products encoded by the placental genes (Table 1). Whereas transcriptional regulators (Fig. 1d) were over-represented among de-regulated (b) embryo genes that were misregulated under gestational diabetic conditions by more than twofold at $p<0.05$. Classification of misregulated (c) placenta genes and (d) embryo genes by molecular function, as indicated by colours

genes in diabetes-exposed embryos [9], we did not find this when comparing diabetic and control placentae (Fig. 1c). Instead, DAVID analysis provided evidence of enrichment of molecules with roles in cell adhesion and receptormediated signalling, with the majority of genes in these categories $(63.2 \%$ and $77.3 \%$, respectively) exhibiting higher expression levels in diabetic placenta than in control. These results implicate processes at the cell surface and signalling as the major pathways affected in diabetic placenta.

Table 1 Genes differentially expressed in diabetic placenta classified by cellular function

\begin{tabular}{|c|c|c|c|}
\hline Functional category & Genes $(n)$ & Percent & Gene symbol \\
\hline $\begin{array}{l}\text { Extracellular matrix, cell adhesion, } \\
\text { cell migration }\end{array}$ & 19 & 12.0 & $\begin{array}{l}\text { Anxa4, Fam19a5, Ceacam15, Cldn23, Cldn3, Emid1, Emp2, Ggt a1, Gpld1, } \\
\text { Igsf11, Lims1, Mmp9, Mpzl2, Pfpl, Pkp2, Pvrl1, Thsd4, Tmem30a,Tmem46 }\end{array}$ \\
\hline $\begin{array}{l}\text { Soluble factors, hormones, ligands, } \\
\text { ligand binding proteins }\end{array}$ & 15 & 9.5 & $\begin{array}{l}\text { Angpt4, Bmp2, Calcb, Cck, Cx3cl1, Fgfbp3, Fstl3, Osm, Prl5a1, Ren1/Ren, Sfrp5, } \\
\text { Tac4, Tgfbi, Thbs1, Thbs2 }\end{array}$ \\
\hline Cell surface receptors & 7 & 4.4 & Chrm1, Csf2rb, Cxcr4, Gpr161, Hhipl1, Ms4a4c, Tnfrsf1b \\
\hline Signal transduction & 12 & 7.6 & Ctnnd2, Dab1, Ehd3, Gnao1, Hipk2, Pctk3, Phip, Prkg2, Rhov, Rock1, Socs3, Sphk1 \\
\hline Transcription factors & 15 & 9.5 & $\begin{array}{l}\text { Ascl2, Atoh8, Cdx2, Creb3l3, E2f2, Fosl2, Foxk2, Hand2, Myb, Nfatc1, Prrx2, } \\
\text { Smad6, Srebf2, Zbtb20, Zfp458 }\end{array}$ \\
\hline DNA-binding/chromatin & 3 & 1.9 & Ddx31, Fancm, Whsc1l1 \\
\hline Metabolism/enzymes & 12 & 7.6 & $\begin{array}{l}\text { Aldh5a1, Car5b, Ckmt1, Cypla1, Eno1, Haghl, Pgk1, Fabp4, Gdpd1, Mgam, } \\
\text { Pank1, Tdo2 }\end{array}$ \\
\hline Lipid metabolism & 3 & 1.9 & $L p l, O s b p$, Star \\
\hline Transporter/channels & 10 & 6.3 & Atpla3, Ccbe1, Itpr2, Kcnk2, Kcnn4, Slc28a3, Slc41a3, Slc6a4, Slco2a1, Tmc5 \\
\hline Protein processing & 12 & 7.6 & Arl15, Cts7, Gcnt3, Il4i1, Lta4h, Pcsk5, Ppm1j, Prosc, Spsb4, Trip12, Trim63, Usp32 \\
\hline Cytoskeleton/microtubules & 7 & 4.4 & Actc1, Ankrd2, Dctn5, Kif18a, Phr1, Synpo2l, Tsga10 \\
\hline Cell cycle/apoptosis & 3 & 1.9 & Ambra1, Incenp, Pacs 2 \\
\hline RNA binding & 2 & 1.3 & Ear11, Sfrs 3 \\
\hline Metal ion homeostasis & 1 & 0.6 & Necab2 \\
\hline Other & 9 & 5.7 & Hjurp, Hspala, Mrps23, Nup88, Rad51c, Rmi1, Sfrs18, Vps11, Vamp5 \\
\hline Unknown & 28 & 17.7 & $\begin{array}{l}\text { Bat2d, Bud31, Ccdc34, Ier5, Klhl5, Prdm10, Sbsn, Sfmbt2, Ttc28, Zdhhc14, AI414330, } \\
\text { AU015536, BC020535, C76555, C80406, D9Ertd115e, LOC100043487, } \\
\text { Rik1500009L16, Rik1810010M01, Rik2610019E17, Rik2610028H07, } \\
\text { Rik5033413D22, Rik5330426P16, Rik6820431F20, Rik9130008F23, } \\
\text { RikB130019D13, RikD130040H23, RikF630043A04 }\end{array}$ \\
\hline Total & 158 & 99.9 & \\
\hline
\end{tabular}

Information was obtained from Gene Ontology annotations and manually annotated with input from MGI and PubMed 
There was little overlap of the genes we identified as misregulated in diabetic placentae at mid-gestation with those reported by others for late gestation. In fact, no genes were found in common with results from a 151 gene custom array applied to ICR outbred mouse strain diabetic placentae at gestational day 17 [21] or from cDNA arrays of E17.5 diabetic placentae from the C57BL/ 6, BALB/c, DBA/1 and CBA/J inbred mouse strains [22]. In human term placentae from pregnancies affected by gestational diabetes, TGFß-induced transcript, thrombospondin 1 and heat shock protein A1A had increased and follistatin-like 3 decreased expression [23], while we saw decreased levels for all four genes. Fos-like antigen 2 and annexin A4 were elevated in an independent study of gestational diabetes mellitus placentae [24], while annexin A4 was decreased in our assays. Apart from strain or species differences, the most likely reason for the dearth of common discoveries is the early time point of our assays. Between E10.5 and E17 in the mouse, and mid-term to term in humans, respectively, the placenta undergoes fundamental changes in gene expression [25] in the maternally and embryo-derived compartments [26]. Thus, even in normal placenta at E10.5, we would expect different sets of genes to be expressed compared with later-stage placenta as surveyed by others. Differential gene expression under conditions of maternal diabetes has also been reported for the yolk sac in rats at E12.5 [27]. The only feature common to our results is increased enolase 1 expression. We therefore conclude that yolk sac and placenta undergo distinct responses to maternal diabetes. Taken together, our results show that, placental gene expression is uniquely altered in diabetic pregnancy in mice as early as E10.5.

Validation of microarray results by quantitative real-time $P C R$ For validation by quantitative real-time PCR, we selected differentially expressed genes with high and moderate expression levels. Placenta samples were from different pregnancies than those assayed in the microarray, thus providing independent biological material from the same diabetes paradigm. Table 2 shows that of 27 genes assayed, 17 exhibited differential expression in the quantitative real-time PCR assay with statistical significance set at $p<0.05$, confirming the microarray results in independent samples ( $n=6$ per group). Thus, quantitative real-time PCR provided independent validation of differential expression between diabetic and control samples for $63 \%$ of the genes tested. It is also noteworthy that the direction of change (increase/decrease) was $100 \%$ consistent between microarray and PCR for the validated genes.

Transcriptional regulation If cohorts of genes in the placenta are dysregulated through shared pathways of transcriptional regulation, one would expect occurrence of common TFBSs in the regulatory regions of these genes. In embryos exposed to hyperglycaemia [9], we previously found over-representation of binding motifs for forkhead box (FOX)O1, FOXO4 and nuclear factor erythroid 2related factor 2 (NRF2), which are involved in the oxidative stress response, and for hypoxia-inducible factor 1 (HIF1), which orchestrates the response to hypoxia. Both oxidative stress and hypoxia have been reported for embryos exposed to diabetes [6]. However, when we analysed promoter sequences $(5 \mathrm{~kb}$ upstream of the transcription start) of diabetes-affected placental genes for the presence of TFBS, we found no enrichment of HIF1 sites. In addition, neither Hifl $\alpha$ expression nor expression of any of its known targets [28] were perturbed in placentae from diabetic dams, providing scant evidence for a hypoxia response in the placenta.

For FOXO1 and FOXO4, putative binding sites were present in 85 and 82 genes, respectively, of 131 placental genes for which results were returned (this accounts for $65 \%$ and $62.6 \%$, respectively, of 131 genes, with 80 genes having both motifs). In addition, there was significant enrichment of these TFBS $\left(-\log _{10} p=6.7809\right.$ and $-\log _{10} p=$ 5.55 , respectively) compared with the entire genome. In placenta, oxidative stress has been associated with activation of nuclear factor $\mathrm{kB}(\mathrm{NF} \kappa \mathrm{B})[29,30]$, and we found TFBS motifs for NFkB-enrichment $\left(-\log _{10} p=5.076\right)$, with 63 genes (48\% of 131) carrying these sites. These results suggest that oxidative stress pathways could be involved in deregulated gene expression in the diabetic placenta.

Functional roles of genes deregulated in diabetic placentae Conceivably, misregulated genes in diabetic placentae could be involved in altered placental function. This proposition is supported by mouse mutant phenotype information (MGI), which is available for 68 of the 158 genes. Of these 68 genes, seven have been shown to play a role in abnormal development of extra-embryonic tissues, uterus and/or the placenta (ESM Table 4 plus references cited there). Knockout of Incenp and Lims 1 affects extraembryonic tissues, and Bmp2 deficiency delays allantois development, while Star deletion is associated with abnormal uterus morphology. The labyrinth is disorganised in mutants that are heterozygous for a mutant $C d x 2$ allele on a $C d x 4$-deficient background. Animals with knockout of Socs 3 and Ascl2 exhibited altered placental morphology in the labyrinth layer, with the latter gene also affecting trophoblast giant cells and spongiotrophoblasts. However, for many other diabetes-affected placental genes, functional annotation is not yet available. Interestingly, the number of genes we identified as altered in diabetic placenta is only a small fraction of the 458 genotypes listed in MGI with placental phenotypes. This strongly suggests that diabetes affects specific pathways in the placenta, such as cell 
Table 2 Validation of microarray results by quantitative RT-PCR

\begin{tabular}{|c|c|c|c|c|c|}
\hline \multicolumn{2}{|c|}{ Gene designation } & \multicolumn{2}{|l|}{ Microarray } & \multicolumn{2}{|c|}{ Real-time PCR } \\
\hline Symbol & Name & Fold change & $p$ value & Fold change & $p$ value \\
\hline \multicolumn{6}{|c|}{ Gene expression significantly changed in RT-PCR } \\
\hline Atoh 8 & Atonal homologue 8 (Drosophila) & 2.17 & 0.0012 & 1.57 & 0.0116 \\
\hline Bmp2 & Bone morphogenetic protein 2 & 2.47 & 0.0003 & 1.36 & 0.0165 \\
\hline Calcb & Calcitonin-related polypeptide, beta & 2.24 & 0.0116 & 1.93 & 0.0015 \\
\hline Cck & Cholecystokinin & 2.91 & $<0.0001$ & 1.71 & 0.0254 \\
\hline Earl1 & Eosinophil-associated, ribonuclease A family, member 11 & 2.74 & 0.0120 & 1.53 & 0.0235 \\
\hline Mmp 9 & Matrix metallopeptidase 9 & 3.28 & 0.0157 & 1.59 & 0.0228 \\
\hline Pcsk5 & Proprotein convertase subtilisin/kexin type 5 & 2.63 & 0.0041 & 1.55 & 0.0028 \\
\hline Pfpl & Pore forming protein-like & 3.23 & 0.0282 & 2.05 & 0.0018 \\
\hline Prl5al & Prolactin family 5 , subfamily a, member 1 & 2.54 & 0.0164 & 1.95 & 0.0081 \\
\hline Slc6a4 & Solute carrier family 6 (neurotransmitter transporter, serotonin), member 4 & 2.61 & 0.0016 & 2.45 & 0.0003 \\
\hline Ankrd2 & Ankyrin repeat domain 2 (stretch responsive muscle) & -2.16 & 0.0003 & -3.03 & 0.0003 \\
\hline Ascl2 & Achaete-scute complex homologue-like 2 (Drosophila) & -1.72 & 0.0148 & -1.66 & 0.0036 \\
\hline Ctsq & Cathepsin Q & -1.73 & 0.0059 & -2.69 & 0.0070 \\
\hline Mpzl2 & Myelin protein zero-like 2 & -1.80 & 0.0007 & -1.29 & 0.0084 \\
\hline Rassf4 & Ras association (ralgds/AF-6) domain family 4 & -1.67 & 0.0135 & -1.52 & 0.0089 \\
\hline$T g f b i$ & Transforming growth factor, beta induced & -1.89 & 0.0003 & -1.56 & 0.0249 \\
\hline$T p b p b$ & Trophoblast specific protein beta & -1.95 & 0.0006 & -2.02 & 0.0094 \\
\hline \multicolumn{6}{|c|}{ Gene expression not significantly changed in RT-PCR } \\
\hline Adamts6 & A disintegrin-like and metallopeptidase with thrombospondin type 1 motif, 6 & 1.54 & 0.0187 & 1.07 & 0.6068 \\
\hline$C s f 2 r b$ & Colony stimulating factor 2 receptor, beta, low-affinity (granulocyte-macrophage) & 2.14 & 0.0040 & 1.30 & 0.1102 \\
\hline Kcnk2 & Potassium channel, subfamily K, member 2 & 2.14 & 0.0031 & 1.02 & 0.8894 \\
\hline Sfrp 5 & Secreted frizzled-related sequence protein 5 & 1.61 & 0.0122 & 1.10 & 0.5603 \\
\hline Slco2al & Solute carrier organic anion transporter family, member $2 \mathrm{a} 1$, prostaglandin transporter & 1.86 & 0.0059 & 1.09 & 0.6437 \\
\hline Smad6 & MAD homologue 6 (Drosophila) & 1.86 & 0.0165 & 1.29 & 0.1631 \\
\hline Thbs 2 & Thrombospondin 2 & 1.93 & 0.0079 & 1.38 & 0.1885 \\
\hline Cyplal & Cytochrome P450, family 1 , subfamily a, polypeptide 1 & 1.92 & 0.0044 & -1.07 & 0.7371 \\
\hline Klhl5 & Kelch-like 5 (Drosophila) & 1.77 & 0.0442 & -1.08 & 0.3275 \\
\hline Thsd4 & Thrombospondin, type I, domain containing 4 & 2.18 & 0.0040 & -1.13 & 0.4449 \\
\hline
\end{tabular}

For each gene, the amplification rate was calculated from the geometric range of the respective reaction, and placentae from independent diabetic dams were compared ( $n=6$ except otherwise indicated) with placentae from independent control pregnancies $(n=6$ except otherwise indicated)

adhesion, growth factor responses and nutrient transport. Collectively, our results are consistent with the notion that misregulation of gene expression compromises development and function of the placenta in diabetic mouse pregnancy.

Expression studies identified abnormalities in the maternal and embryonic compartments of the diabetic placenta To identify the cell types with altered gene expression, we performed in situ hybridisation studies. Figure 2 depicts hybridisation patterns in normal E10.5 placenta for selected genes for which placental expression has not been described previously. Mmp9 was expressed in a few cells of the junctional zone, thrombospondin-domain 4 exhibited a diffuse pattern, and in the decidual compartment we detected Sfrp5 and Rik9130008F23. Expression of all these genes was increased under conditions of diabetes (ESM Table 2). Using microarray and RT-PCR, we also found decreased expression in diabetic placentae of Ascl2 (Fig. 3a-c) and ankyrin-repeat-domain 2 (Fig. 3d-f), which is expressed in the same region, encompassing junctional layer and labyrinth.

Particularly noteworthy are increased hybridisation signals for the Slc6a4 (serotonin transporter), Cyplal, calcitonin-related-peptide B and cholecystokinin genes in diabetic placenta (Fig. 3g-r). In all four cases, the number of cells expressing the respective transcript was increased. The identity of cells expressing Slc6a4 (Fig. 3g-i) adjacent to the junctional layer remains to be determined; a weak signal was also detected in the labyrinth. Cypla1 (Fig. 3j-1) 
Fig. 2 Expression of selected misregulated genes in normal midterm placenta. a Schematic representation of mouse mid-gestation placenta. b Visualisation of maternal and embryonic compartments of the placenta on histological sections by staining with periodic acid-Schiff's reagent and Methyl green. $\mathbf{c}-\mathbf{f}$ In situ hybridisation of selected genes to normal placenta at E10.5

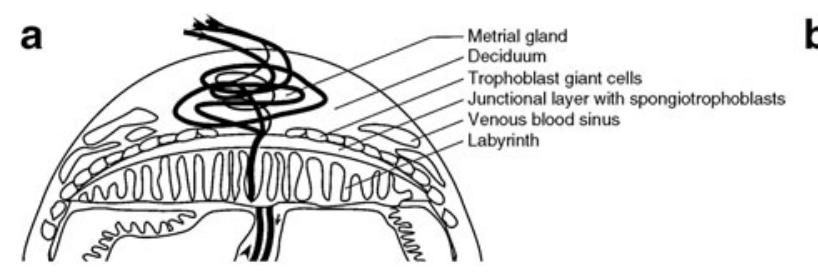

b

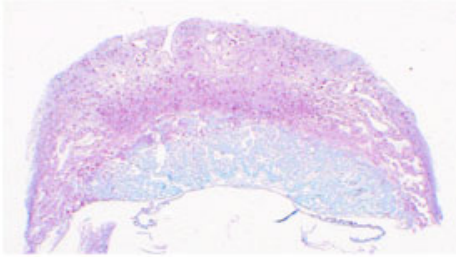

C

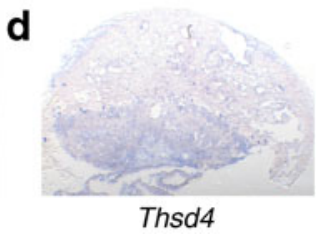

e

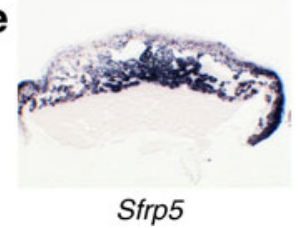

f

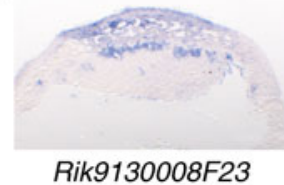

Fig. 3 Misregulated gene expression in diabetic placenta. In situ hybridisation was performed in parallel for sections from control (a, d, $\mathbf{g}, \mathbf{j}$, $\mathbf{m}, \mathbf{p})$ and diabetic $(\mathbf{b}, \mathbf{e}, \mathbf{h}, \mathbf{k}, \mathbf{n}$, q) placentae at gestational day E10.5. For each placenta, ten sections were hybridised to the same probe preparation, and for each gene at least two independent experiments were performed with two different placentae per modality. $(\mathbf{a}-\mathbf{c})$ Ascl2; (d-f) Ankrd2; (g-i) Slc6a4; (j-l) Cypla1; (m-o) Calcrb; (p-r) Cck. Magnified images of (c) Ascl2 and (f) Ankrd2 expression in normal placenta. Magnified image of (i) Slc6a4, (1) Cypla1, (o) Calcrb and (r) Cck expression in diabetic placentae, respectively. No hybridisation was observed with a probe for Gastrin, which shares amino acid identity with $C c k$
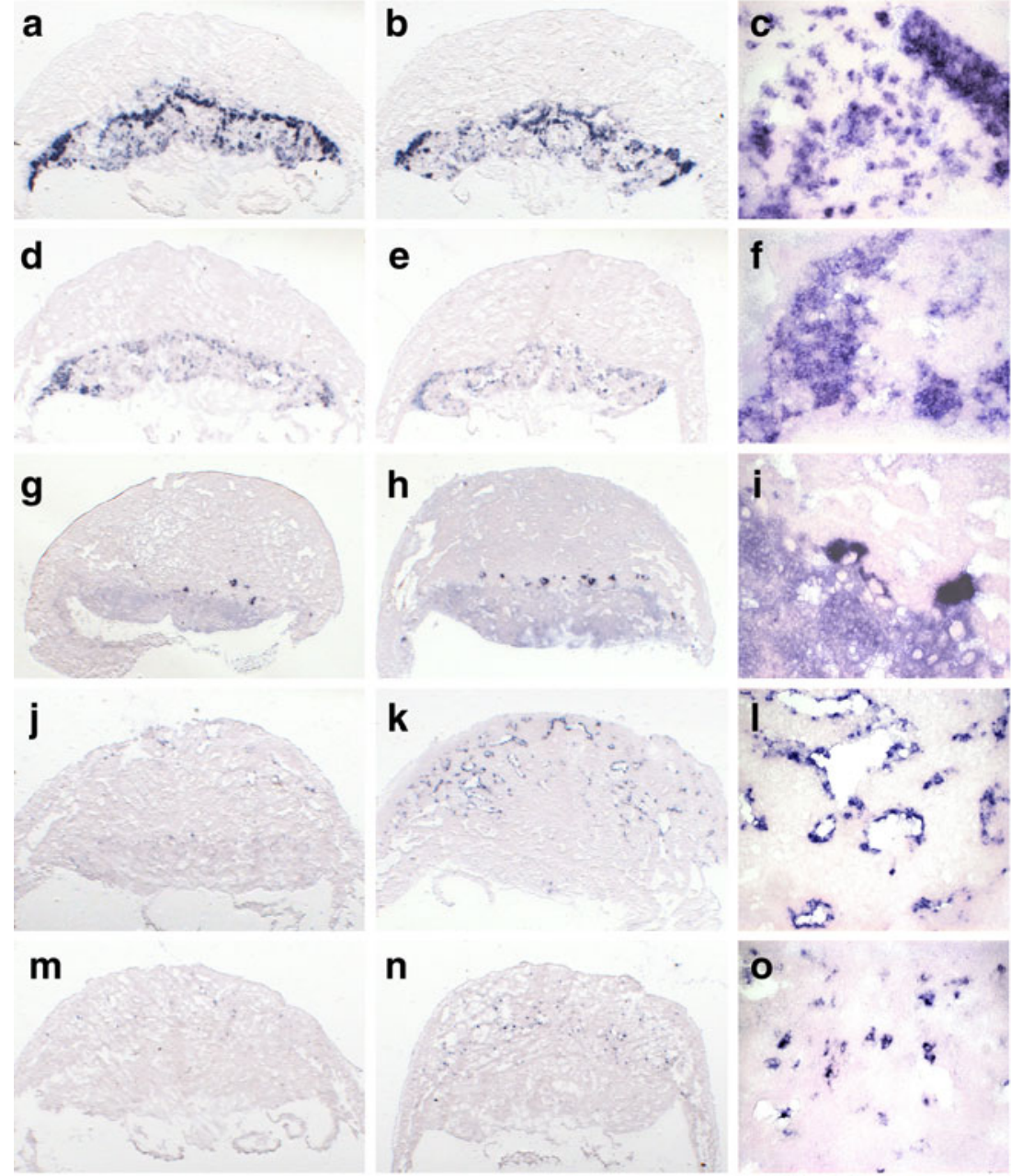

p
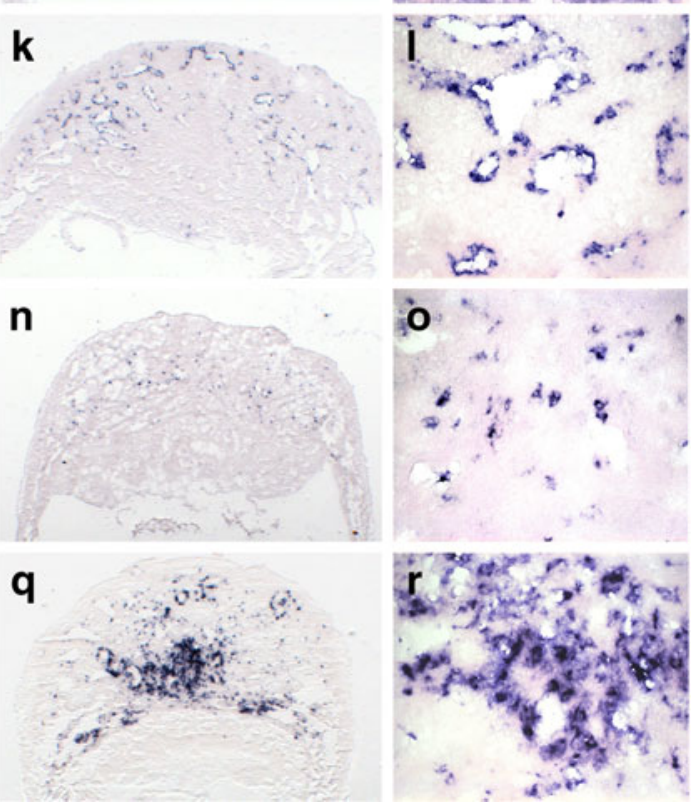
was expressed in cells lining the decidual blood sinuses, consistent with a report that found the same localisation after administration of a toxic compound [31]. Elevated Cyplal expression and activity in placenta is a wellknown indicator of exposure to xenogenic substances, most prominently linked to maternal cigarette smoking [32]. In diabetic placentae, the number of Cypla1expressing cells was increased (Fig. 3k). Although in human diabetic pregnancies, P450 enzyme activities were not elevated [33] in term placenta, our results from midgestation diabetic mice support the notion that elevated Cyplal expression may have a physiological role in diabetic pregnancies, possibly in the removal of undesirable metabolites.

Calcitonin-related-peptide $B$ gene expression levels were also increased in diabetic placenta (Fig. 3n), this being again associated with an increase in the number of positive cells, which were dispersed in the decidual part of the placenta (Fig. 3o), and whose identity and physiological role remain to be clarified. Calcitonin-related peptides are known to be potent vasodilators in placenta [34], and increased blood flow to implantation sites has been documented in streptozotocin-induced diabetic rats [35]. It is thus possible that increased Calcitonin-related peptide B levels could be involved in placental complications in diabetic pregnancies.

Robust induction by maternal diabetes was observed for the gene encoding cholecystokinin, a gastrointestinal peptide and neurotransmitter. In diabetic placentae, the signal for $C c k$ mRNA was clearly elevated (Fig. 3q); again, this was associated with a larger number of $C c k$-expressing cells. The identity of these cells in the metrial region (Fig. 3r) is currently undetermined; a subset is CD45-positive (data not shown), indicating a haematopoietic origin.

Taken together, our studies demonstrate that maternal diabetes alters gene expression in embryonic and maternal compartments of the placenta, including several genes not previously implicated in diabetic pregnancies. In all cases where increased expression was present, this was linked to a greater number of cells expressing the respective gene, demonstrating that the cellular composition of the placenta is altered in diabetic pregnancy.

Reduced junctional zone and labyrinth size in diabetic placentae Histological analysis confirmed the overall smaller sizes of diabetic placentae (Fig. 4) at E15.5 and E18.5. These results are consistent with reports of lower weights for diabetic placentae at E17.5 [22] and E18.5 [36], and with a systematic study throughout pregnancy from our own laboratory (data not shown).

The junctional zone was reduced in diabetic placentae at E15.5 (Fig. 4a-d) and the labyrinth was smaller at E18.5
(Fig. 4e, f). To identify the affected cell types, we performed in situ hybridisation on adjacent sections using a probe specific for the gene encoding the spongiotrophoblastexpressed marker, trophoblast-specific protein alpha (TPBPA) [37]. Since Tpbpa mRNA was expressed in spongiotrophoblasts in the junctional layer (Fig. 5), this allowed us to measure the relative areas of the placenta occupied by junctional layer and labyrinth as a proxy for size of these cellular compartments (Fig. 4i-1). While the relative area for the labyrinth did not differ at E15.5, the area occupied by a strong Tpbpa signal relative to the size of the whole placenta and to the labyrinth was reduced in diabetic placentae (Fig. 4j). However, by E18.8 size of the labyrinth was significantly decreased in diabetic placentae (Fig. 4f, k), while the area fraction for the junctional layer relative to whole placenta or labyrinth was not significantly altered. A reduction of labyrinth size has also been reported for diabetic mouse placentae at E17.5 [22]. Taken together, our data from two different time points indicate that growth of the junctional layer and the labyrinth are affected at temporally distinct phases by maternal diabetes.

Impaired spongiotrophoblast development has been reported for mutants deficient in the genes encoding the transcription factors achaete-scute complex homologue 2 (ASCL2), Hif1 $\alpha$, HOP homeobox or retinoid X receptor (RXR) $\alpha / \beta$ combined (ESM Table 4), as well as for transcriptional co-regulators $\mathrm{Cbp} / \mathrm{p} 300$-interacting transactivator with Glu/Asp-rich carboxy-terminal domain 2 (CITED2) and alkylation repair $B$ homologue 1 (ALKBH1), and for loss of imprinting in the Dnmt3L (also known as Dnmt3l) mutant. Reduced spongiotrophoblast growth was associated with expansion of trophoblast giant cells at mid-gestation [38] and reduction of giant and glycogen cells at E14.5/15.5 [39, 40]. Where diminished labyrinth size was reported for late pregnancy stages, it was interpreted as secondary to the spongiotrophoblast defect $[41,42]$. We therefore consider it likely that the reduced labyrinth in diabetic placentae could be a consequence of impaired spongiotrophoblast growth. Where investigated, reduced spongiotrophoblast growth in mutants was associated with reduced $\mathrm{Ascl} 2$ expression at mid-gestation (ESM Table 4), which we also found in diabetic placentae. Collectively, this evidence supports the conclusion that the spongiotrophoblast deficiency in diabetic placentae is the result of aberrant gene expression at mid-gestation.

Conceivably, the thickness of the spongiotrophoblast layer could also be influenced by cell proliferation. In Senp 2 mutants, reduction of the spongiotrophoblast layer was associated with delayed cell cycle progression [43]. Mice transgenic for cathepsin 7 also display reduced spongiotrophoblast layer by E12.5, attributed to slower progression through late $\mathrm{G} 2$ or M phases of the cell cycle 

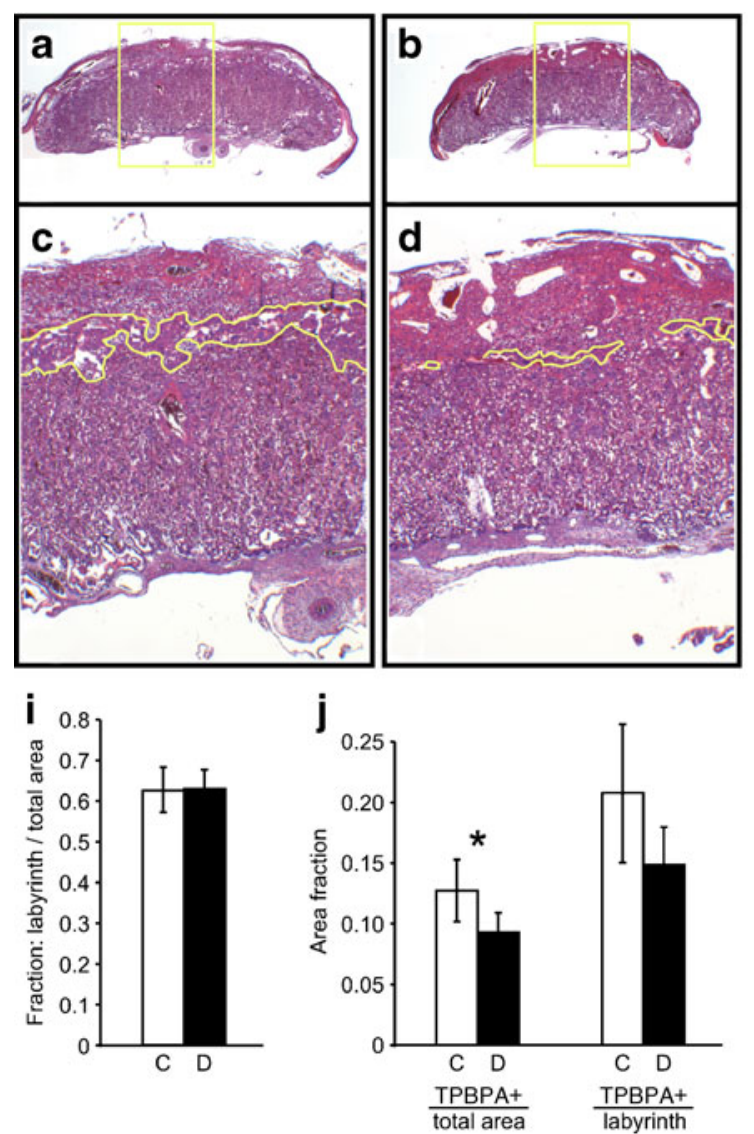

Fig. 4 Histological analysis of control and diabetic placentae. Paraffin-embedded sections were stained with haematoxylin-eosin, and at least ten sections were analysed for each individual placenta. Sections from (a) control and (b) diabetic placentae at E15.5, with respective magnifications $(\mathbf{c}, \mathbf{d})$ of areas in yellow frame $(\mathbf{a}, \mathbf{b})$. Sections from (e) control and (f) diabetic placentae with $(\mathbf{g}, \mathbf{h})$ magnifications as above (a-d) at E18.5. Green double arrows mark

[44]. The possibility that growth factor signalling may be affected by diabetes is suggested by reduced expression of Pappa2, which regulates IGF2 availability (data not shown).

Aberrant spongiotrophoblast differentiation in diabetic placentae Unexpectedly, Tpbpa signal was also detected within the labyrinth of diabetic placentae. At E15.5, numerous clusters of Tpbpa+cells were present (Fig. 5b, e), with lower incidence at E18.5 (Fig. 5h, k). This suggests that diabetic conditions cause ectopic differentiation of cells towards a spongiotrophoblast cell fate, ectopic proliferation of spongiotrophoblast-like cells in the labyrinthine area or aberrant migration into the labyrinth. In normal placentae, some clusters of Tpbpa+cells were observed in the labyrinth at E15.5, but very few at E18.5 (Fig. 5m, o), indicating that such cells are normally either eliminated or migrate out of the labyrinth. In the diabetic placentae, the ectopic Tpbpa+clusters were significantly larger (Fig. 5n)
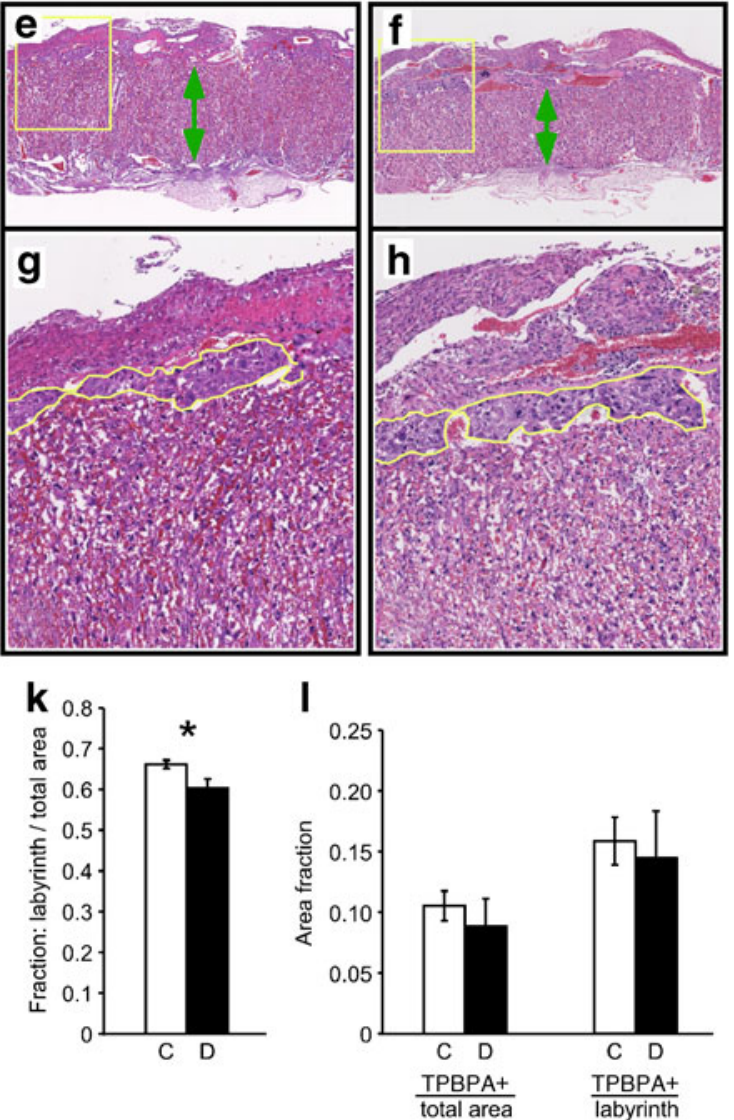

the thickness of the labyrinth. i-l Quantitative analyses of compartment size was facilitated by Tpbpa staining of adjacent sections (see Fig. 5 and text for details). Results are given as fraction of section area occupied by labyrinth (i, $\mathbf{k})$ and as fraction of area occupied by Tpbpa+cells in relation to the area of the entire section and the labyrinth area $(\mathbf{j}, \mathbf{l}) .{ }^{*} p<0.05$. C, control; $\mathrm{D}$, diabetic

and persisted to E18.5. The mislocalisation of these cells outside the spongiotrophoblast layer might explain its reduced thickness. Interestingly, Yu et al. [22] reported moderate enlargement of the junctional layer in E17.5 diabetic mouse placentae, based on isolectinB4 staining, but they included glycogen-containing cells in their measurements. These cells, which display light cytoplasms on haematoxylin-eosin-stained sections [45], exhibit weak Tpbpa labelling (Fig. 5e, f) and were excluded from our analysis because they differ in histological appearance from the cells with the strongest Tpbpa signal at E15.5 (Fig. 5e, f) and at E18.5. The precise relationship of the ectopic clusters in the labyrinth to trophoblast progenitors, spongiotrophoblasts [46] and glycogen cells [45] remains to be defined. The persistence of large clusters of inappropriately differentiated cells may affect the functional integrity of the labyrinth and the vasculature, a possibility that deserves further investigation. Taken together, our results 

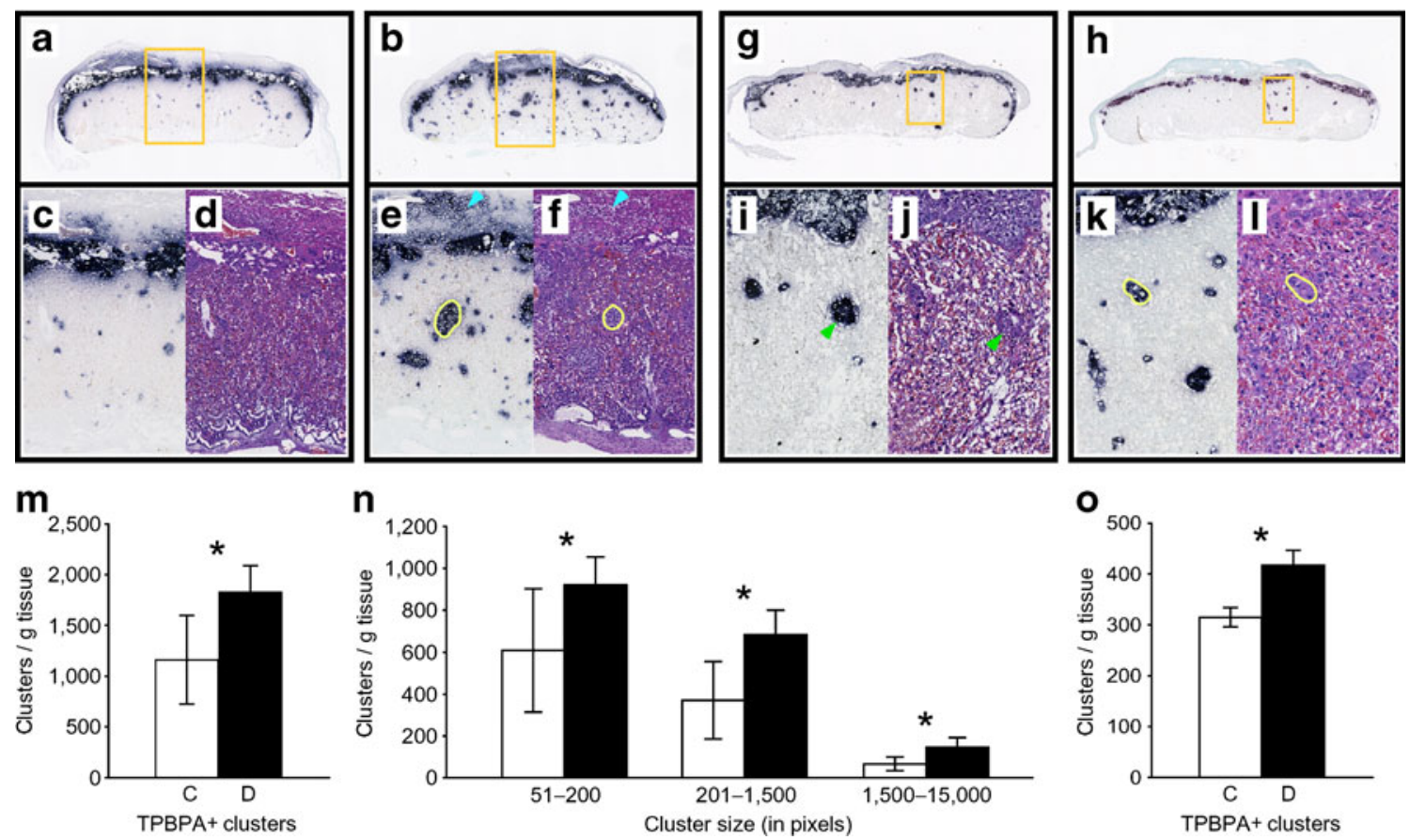

Fig. 5 Aberrant spongiotrophoblast differentiation in diabetic mouse placenta. Ectopic localisation of spongiotrophoblast-like cells was detected by in situ hybridisation with a probe for Tpbpa. Sections from (a) control and (b) diabetic placentae at E15.5, with respective (c, e) magnifications and (h) diabetic placentae at E18.5, with (i, k) magnification and $(\mathbf{j}, \mathbf{l})$ staining as in $\mathbf{a}-\mathbf{f}$. Yellow circles highlight corresponding clusters in diabetic placentae at (e, f) E15.5 and $(\mathbf{k}, \mathbf{l})$ E18.5. Light blue arrowheads (e, f) mark presumed glycogencontaining cells with weak Tpbpa signal. These cells display different histological appearance from the cells in typical clusters in the labyrinth (green arrowheads; i, j). Clusters in the labyrinth were counted on the basis of pixel size for contiguous hybridisation signal greater than 30. Diabetic placentae at (m) E15.5 and (o) E18.5 displayed significantly increased number of clusters (note the different scale of the $y$-axis in $\mathbf{m}$ compared with $\mathbf{o}$ ). $\mathbf{n}$ In diabetic placentae at E15.5, sizes of clusters were also significantly larger, both when the data were classified in three bins and in ten bins (not shown) of increasing size along non-linear scale. ${ }^{*} p<0.05$. C, control; D, diabetic show that maternal diabetes impairs growth and alters spongiotrophoblast differentiation in the placenta, which is likely to affect signalling pathways important for placental growth and may have a detrimental effect on nutrient supply to the embryo.

Mislocalisation of spongiotrophoblast cells to the labyrinth has been reported in only a few instances to date. 'Islands of SP-like cells' [47] in the labyrinth were detected with targeted disruption of the genes encoding haemoxygenase 1, prolyl hydroxylase domain 2 and protocadherin 12, and aberrant expression of the imprinted gene Phlda2 in mice (ESM Table 4). Effects on labyrinth size were not consistent, but in the latter, fetal growth was delayed, resulting in a $13 \%$ smaller size by E18.5. These results implicate ectopic spongiotrophoblast differentiation in intrauterine growth reduction and support the intriguing possibility that the reduced spongiotrophoblast layer and ectopic spongiotrophoblast localisation in diabetic placentae could be responsible for the reduced embryonic growth we previously observed [10] at later stages of mouse diabetic pregnancies.
Finally, although spongiotrophoblasts originate from extra-embryonic progenitors, their differentiation may be influenced by maternal factors. For example, the spongiotrophoblast layer was reduced in mutants with uterusspecific deletion of the Nr2f2 gene (encoding COUPTFII), demonstrating a maternal effect on spongiotrophoblast growth [48]. Given that we found altered cell composition in the decidual compartment of diabetic placentae, it will be interesting to investigate which role specific decidual cell types have in spongiotrophoblast differentiation and placenta growth. Collectively, our results show that maternal diabetes causes misregulation of distinct gene repertoires in embryo and placenta, bringing about developmental defects, intrauterine growth reduction and abnormal placental cell differentiation, respectively. Perturbed cellular interactions in the diabetic placenta and subsequent impairment of fetal growth could be the underlying cause for life-long elevated disease risk for mother and offspring of a pregnancy complicated by diabetes. 
Acknowledgements We are grateful for expert technical assistance by J. MacGowan, who performed the animal work and dissections. This work was funded in part through RO1-HD34706 to C. Kappen with a supplement to G. Pavlinkova, and through RO1-HD055528 to J. M. Salbaum with a supplement to N. Arbour Delahaye, as well as through the Peggy M. Pennington Cole Chair for Maternal Biology (C. Kappen). Histological investigations made use of the Cell Biology and Bioimaging Core Facility at Pennington, and PCR assays were performed in the Genomics Core Facility (both facilities are supported through COBRE P20RR021945 and CNRU P30DK072476). The Comparative Biology Core Facilities received support through G20RR024838.

Duality of interest The authors declare that there is no duality of interest associated with this manuscript.

\section{References}

1. Kucera J (1971) Rate and type of congenital anomalies among offspring of diabetic women. J Reprod Med 7:73-82

2. Martinez-Frias ML (1994) Epidemiological analysis of outcomes of pregnancy in diabetic mothers: identification of the most characteristic and most frequent congenital anomalies. Am J Med Genet 51:108-113

3. Mills JL (1982) Malformations in infants of diabetic mothers. Teratology 25:385-394

4. Plagemann A (2004) 'Fetal programming' and 'functional teratogenesis': on epigenetic mechanisms and prevention of perinatally acquired lasting health risks. J Perinat Med 32:297-305

5. Simeoni U, Barker DJ (2009) Offspring of diabetic pregnancy: long-term outcomes. Semin Fetal Neonatal Med 14:119-124

6. Eriksson UJ (2009) Congenital anomalies in diabetic pregnancy. Semin Fetal Neonatal Med 14:85-93

7. Phelan SA, Ito M, Loeken MR (1997) Neural tube defects in embryos of diabetic mice: role of the Pax-3 gene and apoptosis. Diabetes 46:1189-1197

8. Yang P, Zhao Z, Reece EA (2007) Involvement of c-Jun Nterminal kinases activation in diabetic embryopathy. Biochem Biophys Res Commun 357:749-754

9. Pavlinkova G, Salbaum JM, Kappen C (2009) Maternal diabetes alters transcriptional programs in the developing embryo. BMC Genomics 10:274

10. Kappen C, Kruger C, MacGowan J, Salbaum JM (2011) Maternal diet modulates the risk for neural tube defects in a mouse model of diabetic pregnancy. Reprod Toxicol 31:41-49

11. Godfrey KM (2002) The role of the placenta in fetal programminga review. Placenta 23(Suppl A):S20-S27

12. Fowden AL, Coan PM, Angiolini E, Burton GJ, Constancia M (2010) Imprinted genes and the epigenetic regulation of placental phenotype. Prog Biophys Mol Biol. doi:10.1016/j.pbiomolbio.2010.11.005

13. Padmanabhan R, Shafiullah M (2001) Intrauterine growth retardation in experimental diabetes: possible role of the placenta. Arch Physiol Biochem 109:260-271

14. Nelson SM, Coan PM, Burton GJ, Lindsay RS (2009) Placental structure in type 1 diabetes: relation to fetal insulin, leptin, and IGF-I. Diabetes 58:2634-2641

15. Godfrey KM, Barker DJ (1995) Maternal nutrition in relation to fetal and placental growth. Eur J Obstet Gynecol Reprod Biol 61:15-22

16. Leach L, Taylor A, Sciota F (2009) Vascular dysfunction in the diabetic placenta: causes and consequences. J Anat 215:69-76

17. Jones CJ, Desoye G (1993) Glycogen distribution in the capillaries of the placental villus in normal, overt and gestational diabetic pregnancy. Placenta 14:505-517
18. Kurtz M, Capobianco E, Martinez N et al (2010) Carbaprostacyclin, a PPARdelta agonist, ameliorates excess lipid accumulation in diabetic rat placentas. Life Sci 86:781-790

19. Hauguel-de Mouzon S, Shafrir E (2001) Carbohydrate and fat metabolism and related hormonal regulation in normal and diabetic placenta. Placenta 22:619-627

20. Salbaum JM, Kappen C (2000) Cloning and expression of Nope, a new mouse gene of the immunoglobulin superfamily related to guidance receptors. Genomics 64:15-23

21. Punareewattana K, Gogal RM Jr, Sharova LV, Ward DL, Holladay SD (2005) Reduced birth defects caused by maternal immune stimulation in diabetic ICR mice: lack of correlation with placental gene expression. Immunol Invest 34:71-89

22. Yu Y, Singh U, Shi W et al (2008) Influence of murine maternal diabetes on placental morphology, gene expression, and function. Arch Physiol Biochem 114:99-110

23. Radaelli T, Varastehpour A, Catalano P, Hauguel-de Mouzon S (2003) Gestational diabetes induces placental genes for chronic stress and inflammatory pathways. Diabetes 52:2951-2958

24. Enquobahrie DA, Williams MA, Qiu C, Meller M, Sorensen TK (2009) Global placental gene expression in gestational diabetes mellitus. Am J Obstet Gynecol 200(206):e201-e213

25. Winn VD, Haimov-Kochman R, Paquet AC et al (2007) Gene expression profiling of the human maternal-fetal interface reveals dramatic changes between midgestation and term. Endocrinology 148:1059-1079

26. Knox K, Baker JC (2008) Genomic evolution of the placenta using co-option and duplication and divergence. Genome Res 18:695-705

27. Reece EA, Ji I, Wu YK, Zhao Z (2006) Characterization of differential gene expression profiles in diabetic embryopathy using DNA microarray analysis. Am J Obstet Gynecol 195:1075-1080

28. Semenza GL (2003) Targeting HIF-1 for cancer therapy. Nat Rev Cancer 3:721-732

29. Cindrova-Davies T, Spasic-Boskovic O, Jauniaux E, CharnockJones DS, Burton GJ (2007) Nuclear factor-kappa B, p38, and stress-activated protein kinase mitogen-activated protein kinase signaling pathways regulate proinflammatory cytokines and apoptosis in human placental explants in response to oxidative stress: effects of antioxidant vitamins. Am J Pathol 170:15111520

30. Coughlan MT, Permezel M, Georgiou HM, Rice GE (2004) Repression of oxidant-induced nuclear factor-kappaB activity mediates placental cytokine responses in gestational diabetes. J Clin Endocrinol Metab 89:3585-3594

31. Dey A, Westphal H, Nebert DW (1989) Cell-specific induction of mouse Cypla1 mRNA during development. Proc Natl Acad Sci USA 86:7446-7450

32. Collier AC, Tingle MD, Paxton JW, Mitchell MD, Keelan JA (2002) Metabolizing enzyme localization and activities in the first trimester human placenta: the effect of maternal and gestational age, smoking and alcohol consumption. Hum Reprod 17:25642572

33. McRobie DJ, Glover DD, Tracy TS (1998) Effects of gestational and overt diabetes on human placental cytochromes P450 and glutathione S-transferase. Drug Metab Dispos 26:367-371

34. Mandsager NT, Brewer AS, Myatt L (1994) Vasodilator effects of parathyroid hormone, parathyroid hormone-related protein, and calcitonin gene-related peptide in the human fetal-placental circulation. J Soc Gynecol Investig 1:19-24

35. Zabihi S, Wentzel P, Eriksson UJ (2008) Altered uterine perfusion is involved in fetal outcome of diabetic rats. Placenta 29:413-421

36. Zabihi S, Wentzel P, Eriksson UJ (2008) Maternal blood glucose levels determine the severity of diabetic embryopathy in mice with different expression of copper-zinc superoxide dismutase (CuZnSOD). Toxicol Sci 105:166-172 
37. Lescisin KR, Varmuza S, Rossant J (1988) Isolation and characterization of a novel trophoblast-specific cDNA in the mouse. Genes Dev 2:1639-1646

38. Arima T, Hata K, Tanaka S et al (2006) Loss of the maternal imprint in Dnmt3 $\mathrm{Lmat}^{-1-}$ mice leads to a differentiation defect in the extraembryonic tissue. Dev Biol 297:361-373

39. Withington SL, Scott AN, Saunders DN et al (2006) Loss of Cited 2 affects trophoblast formation and vascularization of the mouse placenta. Dev Biol 294:67-82

40. Pan Z, Sikandar S, Witherspoon M et al (2008) Impaired placental trophoblast lineage differentiation in Alkbh1(-/-) mice. Dev Dyn 237:316-327

41. Tanaka M, Gertsenstein M, Rossant J, Nagy A (1997) Mash2 acts cell autonomously in mouse spongiotrophoblast development. Dev Biol 190:55-65

42. Guillemot F, Nagy A, Auerbach A, Rossant J, Joyner AL (1994) Essential role of Mash-2 in extraembryonic development. Nature 371:333-336
43. Chiu SY, Asai N, Costantini F, Hsu W (2008) SUMO-specific protease 2 is essential for modulating p53-Mdm2 in development of trophoblast stem cell niches and lineages. PLoS Biol 6:e310

44. Screen M, Dean W, Cross JC, Hemberger M (2008) Cathepsin proteases have distinct roles in trophoblast function and vascular remodelling. Development 135:3311-3320

45. Coan PM, Conroy N, Burton GJ, Ferguson-Smith AC (2006) Origin and characteristics of glycogen cells in the developing murine placenta. Dev Dyn 235:3280-3294

46. Simmons DG, Cross JC (2005) Determinants of trophoblast lineage and cell subtype specification in the mouse placenta. Dev Biol 284:12-24

47. Zhao H, Wong RJ, Kalish FS, Nayak NR, Stevenson DK (2009) Effect of heme oxygenase-1 deficiency on placental development. Placenta 30:861-868

48. Petit FG, Jamin SP, Kurihara I et al (2007) Deletion of the orphan nuclear receptor COUP-TFII in uterus leads to placental deficiency. Proc Natl Acad Sci USA 104:6293-6298 